Forthcoming in The British Journal for the Philosophy of Science:

\title{
How to be a Relativistic Spacetime State Realist
}

\author{
Noel Swanson*
}

February 8, 2018

\begin{abstract}
According to spacetime state realism (SSR), the fundamental ontology of a quantum mechanical world consists of a state-valued field evolving in 4dimensional spacetime. One chief advantage it claims over rival wavefunction realist views is its natural compatibility with relativistic quantum field theory (QFT). I argue that the original density operator formulation of SSR cannot be extended to QFTs where the local observables form type III von Neumann algebras. Instead, I propose a new formulation of SSR in terms of a presheaf of local statespaces dual to the net of local observables studied by algebraic QFT.
\end{abstract}

\section{Contents}

1 Introduction $\quad 2$

2 Spacetime State Realism in QFT 5

3 Entanglement and the Type III Property 11

4 Statespace Axioms for QFT 17

5 Discussion 23

*Department of Philosophy, University of Delaware, 24 Kent Way, Newark, DE 19716, USA, nswanson@udel.edu 


\section{Introduction}

There is growing belief that any viable solution to the measurement problem will require treating the quantum state as a genuine physical object rather than an indirect representational tool. This has prompted the development of numerous wavefunction realist views, according to which, the fundamental ontology of a quantum mechanical world consists of a $\mathbb{C}$-valued field (the wavefunction) evolving in a high-dimensional space (usually the configuration space of a non-relativistic many-particle system). Ordinary 4-dimensional spacetime and the objects bumping around inside of it are emergent structures that need to be explained in terms of this high-dimensional alien landscape.

In their 2010 paper, "Quantum Mechanics on Spacetime I: Spacetime State Realism" (BJPS 61, 697-727), David Wallace and Christopher Timpson develop an altogether different kind of view which similarly aims to "take the quantum state seriously" (p. 698). ${ }^{1}$ According to spacetime state realism (SSR), the fundamental ontology of a quantum mechanical world consists of a state-valued field evolving in 4-dimensional spacetime. Each spacetime region is associated with a local Hilbert space whose density operators represent the possible values of the field in that region. Much as in classical field theories, these field values are interpreted as characterizing the intrinsic, local properties of the region.

Unlike wavefunction realism, SSR treats spacetime as the arena in which physics unfolds. Objects like particles and cats are still emergent entities, patterns in the state-valued field, however the defender of SSR does not need to explain the emergence of spacetime itself. This might strike us as a decisive advantage, yet Wallace and Timpson demur. Instead, they think that the primary advantage of SSR is its extendability to relativistic quantum field theory (QFT). SSR, they contend, has an especially simple, elegant relativistic formulation, while there are a number of technical and conceptual pitfalls hampering relativistic extensions of wavefunction

\footnotetext{
${ }^{1}$ See Wallace (2012), Ch. 8 for further development of the view. Arntzenius (2012), Ch. 3.13, Lewis (2013), Baker (2016), and Ismael and Schaffer (2016) represent early critical discussions.
} 
realism. ${ }^{2}$

The reality is not so cut-and-dried. SSR faces its own foundational challenge in the relativistic domain which threatens to undermine this advantage. In standard formulations of QFT, the local states are pieces of derivative structure defined in terms of the global state and the local observable algebras. Self-adjoint elements of these algebras are usually interpreted as representing localized quantities like mass, spin, and charge. From the standpoint of SSR, however, the local observables are not part of the ontology at all. They are merely auxiliary mathematical tools. But if this is the case, we cannot use the concept of local observables to define the local states. Rather, we need a way to formulate QFT that treats the field of local states as fundamental and defines all other structures in terms of it.

In other work $(2006,2012)$, Wallace suggests that such a state-first formulation of QFT is possible. In this paper, I explore the viability of Wallace's proposal and find it wanting. His idea can only get off the ground if the local observable algebras are type I von Neumann algebras familiar from non-relativistic quantum theory. Unfortunately, results from constructive and algebraic QFT indicate that in order to consistently unify relativity and quantum mechanics, the local algebras need to be type III von Neumann algebras. Physically, this reflects the high degree of entanglement exhibited by the QFT vacuum and similar global states. Because these states are so highly entangled, the procedure that Wallace sketches for decomposing the global state into a field of local states falls apart at the hinges, leaving relativistic SSR without a coherent mathematical foundation.

Ultimately we can fix this problem, but it requires a much more radical overhaul of standard QFT than anything Wallace and Timpson suggest. It is well-known that every operator algebra has a dual object of sorts, its statespace, consisting of all positive, normalized, $\mathbb{C}$-valued linear functionals over the algebra. It is less well-known how to make this duality precise - a deep reconstruction theorem due to Alfsen, Hanche-Olsen, and Shultz (1980) proves that given a certain orientation

\footnotetext{
${ }^{2}$ See Wallace and Timpson (2010), $\S 4$ and $\S 8$, as well as Wallace (2012), p. 304-5 and 316-317 for further discussion.
} 
structure on the statespace, we can recover the algebra. Thus all of the physical information contained in the collection of local observables can in principle be encoded in the dual collection of local, oriented statespaces. To complete the picture, we need a set of axioms for the collection of local statespaces that mirror the usual axioms of algebraic or constructive QFT. ${ }^{3}$

The development of such axioms is an involved mathematical project that will be the subject of future work. ${ }^{4}$ The aim of this paper is to clearly articulate the foundational challenge SSR faces in the relativistic domain and to argue that the new statespace formalism represents the best path forward for developing a QFT-compatible version of the view. In the next section, I review Wallace and Timpson's original density operator proposal for relativistic SSR. In $\S 3$, I argue that the type III character of local algebras in QFT poses a serious challenge for their view. A pair of no-go lemmas shows why easy fixes will not work. In $\S 4, \mathrm{I}$ outline my alternative proposal and sketch some ideas for statespace axioms that mirror the Haag-Kastler axioms for algebraic QFT. Finally, in $§ 5$, I discuss how the new statespace approach can help shed light on the metaphysics of SSR and respond to some recent criticisms of the view that turn on misunderstandings of the original density operator formulation. (Apart from this limited defense, the goal is not to provide a general argument in favor of SSR, nor is it to argue that the statespace formalism is preferable to standard algebraic QFT.)

\footnotetext{
${ }^{3}$ The idea of studying QFT via a presheaf of statespaces can be traced back to unpublished work of John Roberts (Haag 1996, p. 326, 131-132, and 141-142). The major difference between Roberts's proposal and the one surveyed here is the use of the AHS-reconstruction theorem and the central notion of an orientation of a $C^{*}$-statespace to capture dual versions of the usual algebraic axioms. Motivated by the problem of constructing particular models of QFT, Roberts did not seek dualized axioms. In addition, his characterization of the presheaf relies on special properties of von Neumann algebras and thus lacks the full generality of algebraic QFT.

${ }^{4}$ Swanson and Halvorson (in preparation).
} 


\section{Spacetime State Realism in QFT}

In the original formulation of SSR, each spacetime region is associated with a local Hilbert space whose density operators correspond to possible field values encoding intrinsic properties of that region. Wallace and Timpson emphasize the parallels between this picture and classical field theories like electrodynamics. The tensor value of the electromagnetic field at a spacetime point represents certain intrinsic properties of that point. The physics are encoded in how these values vary across spacetime. Similarly, in SSR the field takes a particular quantum state as its value in a region. The physics are encoded in how these field values vary across spacetime, in the mosaic of local states.

In classical theories, specifying the field value at each point in a spacetime region suffices to fix the field configuration in that region. Due to quantum entanglement, this is not true in SSR. If the field in region $O_{1}$ is entangled with the field in region $O_{2}$, specifying the state of $O_{1}$ and $O_{2}$ will not fix the state of the joint region $\mathrm{O}_{1} \cup \mathrm{O}_{2}$. Consequently, in order to specify the global field configuration one needs to provide a field value for every region, including the entirety of spacetime itself. This is known as the non-separability of the quantum state. According to SSR, non-separability reflects the presence of non-local relations between regions which do not supervene on the intrinsic properties of those regions.

Wallace (2013) observes that the mathematical signature of non-separability is the appearance of tensor product structure in quantum theory. If $\mathcal{H}_{1}$ and $\mathcal{H}_{2}$ are the local Hilbert spaces associated with causally independent regions $O_{1}$ and $O_{2}$, the statespace of the joint system $O_{1} \cup O_{2}$ is the tensor product $\mathcal{H}_{1} \otimes \mathcal{H}_{2}$. This Hilbert space includes density operators that cannot be factored into products of density operators on $\mathcal{H}_{1}$ and $\mathcal{H}_{2}$, representing entangled states across $O_{1}$ and $\mathrm{O}_{2}$. In contrast, in classical theories the statespace of a joint system is given by

a Cartesian product. As a result every state of the joint system is a product of subsystem states.

For non-relativistic particle theories we can combine these ideas into a simple mathematical picture. Galilean spacetime is naturally foliated into spatial 
hypersurfaces of constant universal time. Given an arbitrary partition of one of these hypersurfaces, each spatial region, $\Delta_{i}$, can be assigned a Hilbert space, $\mathcal{H}_{i}$, whose density operators represent the possible particle states in that region. ${ }^{5}$ The Hilbert space for the entire hypersurface is given by the tensor product of these local Hilbert spaces:

$$
\mathcal{H}=\bigotimes_{i} \mathcal{H}_{i}
$$

If the state of the universe at time $t$ is $\rho$, the local state in region $\Delta_{i}$ is given by

$$
\rho\left(\Delta_{i}\right)=\operatorname{Tr}_{j \neq i} \rho
$$

the partial trace of $\rho$ over all local Hilbert spaces except $\mathcal{H}_{i}$. The local state is a density operator acting on $\mathcal{H}_{i}$.

Extending this scheme to relativistic theories appears straightforward. Wallace and Timpson observe:

Things become simpler still when we move to full quantum field theory. In the algebraic formulation of QFT, we associate to each spacetime region $O$ a $C^{*}$-algebra $\mathfrak{A}(O)$ of operators, representing the dynamical variables associated to region $O$. A state $\rho(O)$ of such a region is a positive linear functional on $\mathfrak{A}(O)$ (often described in rather instrumentalist terms as giving the expectation value of each element of $\mathfrak{A}(O))$ and by the Gelfand-Naimark-Segal (GNS) construction we can associate $\rho(O)$ with a state in a Hilbert space $\mathcal{H}_{O}$, and represent $\mathfrak{A}(O)$ as an algebra of operators on $\mathcal{H}_{O}[\ldots] \mathcal{H}_{O}$ can then be taken as the Hilbert space of the field in region $O$. If preferred, one can even remain at the more abstract level, forego the representation theorems and just take the $C^{*}$-algebraic state itself as denoting the properties of a region. (p. $711-12)^{6}$

\footnotetext{
${ }^{5}$ Wallace and Timpson define $\mathcal{H}_{i}:=\mathcal{F}\left(\mathcal{K}_{i}\right)$, where $\mathcal{F}\left(\mathcal{K}_{i}\right)$ is the Fock space built out of copies of the Hilbert space $\mathcal{K}_{i}$, consisting of single-particle wavefunctions with support in region $\Delta_{i}$.

${ }^{6}$ The original notation has been modified for consistency with the notation of this paper.
} 
There is something rather odd, though, about the scattershot combination of ideas expressed in this paragraph. Algebraic QFT presents us with a global state plus a net of local observable algebras. By taking the restriction of the global state, $\rho$, on a local algebra,

$$
\rho(O):=\left.\rho\right|_{\mathfrak{A}(O)}
$$

we are able to define a unique local state associated with each region. The problem is that this concept of a "local state" is defined in terms of another primitive concept, a "local observable algebra." But according to SSR, the observables are not part of the ontology at all! As Wallace and Timpson explain,

To every different quantum state corresponds a different concrete way the world is. For Everett and for some readings of dynamical-collapse theories, the quantum state (perhaps together with some background space or spacetime) gives the only part of the ontology. (p. 703)

This viewpoint is reinforced by Wallace's critique of Deutsche and Hayden's alternative operator-valued field ontology:

[...] different states of affairs are represented by different state vectors. From this perspective, the operators - time-dependent or not - do not directly give the state of the system; they are simply part of the mathematical machinery which breaks the symmetries of Hilbert space and allows different rays in Hilbert space to represent different states of affairs. (Wallace 2012, p. 320)

But if the local observables are just mathematical machinery, the various definitional procedures Wallace and Timpson discuss become physically opaque. We need to know what connects a particular state to a particular region. Why is $\rho(O)$ the local state for region $O$ ? We cannot point to the fact that $\rho(O)$ encodes the expectation values for the observables localized in $O$, since according to SSR the observables do not correspond to anything physical. We need a way to characterize $\rho(O)$ and its relationship to $O$ that is independent of the mathematical crutch of local observables. 
Although Wallace and Timpson never directly address this issue, Wallace's comments elsewhere point towards a possible resolution:

In the so-called 'algebraic' description of QFT, an algebra of operators is associated to each spatial region, so that the operators associated with region $O$ are intended to represent observables localized in $O$. At least formally, we can regard this as equivalent to decomposing the Hilbert space into quotient spaces, each one representing the quantum state of a different spatial region [...] When a QFT is specified in this way, there is no need to say which operator at $O$ corresponds to which observable property of $O$ - once the localization properties of all the operators are known, the quantum system is sufficiently structured to permit physics to be done, and in particular, to understand the emergence of particles. (Wallace 2012, p. 15)

Wallace (2006) gives a clearer idea of the formal equivalence alluded to here. In all standard formulations of QFT, we begin by assigning local operators (either unbounded field operators or bounded observable operators) to spacetime regions. Choosing a spacelike hypersurface and a partition, as before, Wallace argues that there is a natural tensor product decomposition of the global Hilbert space $\mathcal{H}=$ $\otimes \mathcal{H}_{i}$ in which the operators localized in $\Delta_{i}$ act trivially on all $\mathcal{H}_{j}$ with $j \neq i$. In the usual picture, this justifies calling $\mathcal{H}_{i}$ the "local statespace" associated with region $\Delta_{i}$. As Wallace emphasizes, however, nothing prevents us from switching the order of definition around. Given a tensor product decomposition $\mathcal{H}=\bigotimes \mathcal{H}_{i}$, where $\mathcal{H}_{i}$ is the local Hilbert space associated with spatial region $\Delta_{i}$, we can define operators localized in $\Delta_{i}$ as those linear transformations on $\mathcal{H}$ which act trivially on all $\mathcal{H}_{j}$ with $j \neq i$. This, he contends, provides a state-first formulation of QFT equivalent to the usual, operator-first perspective.

This equivalence thesis is needed in order to make sense of Wallace and Timpson's proposal for relativistic SSR. Despite the widespread use of local operators as part of the mathematical machinery of QFT, we can do without them in principle. All of the information encoded in the system of local field operators and observable 
algebras can be captured by tensor product decompositions of the global Hilbert space. According to SSR, these decompositions directly encode the physical relationship between local states and regions.

As it stands, the thesis is a bit rough around the edges. Given the arbitrariness of choosing a foliation, it would be far more elegant to have a formulation of SSR that utilizes spatiotemporal rather than spatial regions. Not only would this render SSR's starting point more similar to existing axiomatic approaches, but various no-go arguments suggest that local fields and observables in interacting QFTs are only well-defined in 4-dimensional regions, making such a generalization apparently necessary. ${ }^{7}$ Another complication concerns the role of the tensor product structure, which on Wallace and Timpson's formulation effectively does double duty. It serves both to identify the local Hilbert spaces and to stitch them together into a single mathematical object whose sections represent field configurations. But the tensor product structure is ill-suited for this second role. In general, we can only expect the joint Hilbert space associated with two regions to be given by a tensor product if the regions are spacelike separated and thus causally independent. ${ }^{8}$ Moreover, if there are an infinite number of local Hilbert spaces, and they each have dimension greater than two, their infinite tensor product will be nonseparable (i.e., the global Hilbert space will lack a countable basis). But all standard frameworks for QFT employ separable global Hilbert spaces, so if we are looking for a similar formulation of SSR, we can only use the tensor product structure to talk about finite collections of spacelike separated regions. ${ }^{9}$ In order to do physics, we will

\footnotetext{
${ }^{7}$ These arguments typically rely on the singular nature of the renormalized perturbative expansion of the dressed fields in interacting QFTs (e.g., Haag 1996, p. 55-57) and are therefore not entirely rigorous. There are some rigorous results that suggest such smearing is necessary, but they are not conclusive. See Halvorson and Müger (2006), §6 for a survey.

${ }^{8}$ On Wallace's picture, given a tensor product $\mathcal{H}_{1} \otimes \mathcal{H}_{2}$ associated with regions $O_{1}$ and $O_{2}$, the corresponding local observables will be elements of the algebras $B\left(\mathcal{H}_{1}\right) \otimes I$ and $I \otimes B\left(\mathcal{H}_{2}\right)$, which mutually commute. In generic models of QFT, however, only the algebras of spacelike separated regions are guaranteed to commute.

${ }^{9} \mathrm{~A}$ formalization of QFT using nonseparable Hilbert spaces and infinite tensor products might be possible, but such a framework would be unlike anything currently on the table (at least from a mathematical perspective). In contrast, the statespace view I develop in $\S 4$ hews closely to standard algebraic QFT. Halvorson and Müger (2006), §6.2.2 discuss some possible physical
} 
need additional information about the relations between different tensor product decompositions.

We can readily modify Wallace's proposal to take these complications into account while preserving the idea that we rely on tensor products to identify and isolate subsystems. Let $O^{\prime}$ denote the spacelike complement of region $O$ (i.e., the set of points spacelike separated from all points in $O$ ). We can view $O^{\prime}$ as the causally independent environment associated with spatiotemporal system $O$. The key idea will be to focus on the collection of all such system-environment decompositions:

Equivalence Thesis. Given a system-environment decomposition of the global Hilbert space,

$$
\mathcal{H}=\mathcal{H}_{O} \otimes \mathcal{H}_{O^{\prime}}
$$

for each region $O$, define the local algebra of observables associated with $O$ as the algebra of bounded linear operators on $\mathcal{H}_{O}$ :

$$
\mathfrak{A}(O):=B\left(\mathcal{H}_{O}\right)
$$

Given this collection of system-environment decompositions, along with some suitable family of mappings between them, we can define a corresponding net of local observable algebras acting on $\mathcal{H}$ that satisfy the Haag-Kastler axioms for algebraic QFT. Moreover, any net satisfying the axioms arises in such a way.

To make this precise, we need to specify the relevant family of mappings encoding relations between different system-environment decompositions. (At a minimum, if $O_{1}$ is a subregion of $O_{2}$, the local Hilbert space $\mathcal{H}_{O_{1}}$ should be identified with a subspace of $\mathcal{H}_{\mathrm{O}_{2}}$.) In addition, we might also hope to show that the reconstructed net of observables is either unique or natural in some sense. In the end it does not matter. The thesis cannot possibly be true in its current formulation. To see why, we will have to delve deeper into the algebraic structure of QFT.

applications of nonseparable Hilbert spaces. 


\section{Entanglement and the Type III Property}

A $C^{*}$-algebra is an abstract collection of operators isomorphic to some subalgebra of $B(\mathcal{H})$. In algebraic QFT, a theory is given by a net of local $C^{*}$-algebras, $\{\mathfrak{A}(O)\}$, satisfying the Haag-Kastler axioms, along with a collection of physically possible global states, $\{\rho\}$, on the quasilocal algebra, $\mathfrak{A}$ (the norm-closed algebra generated by all local algebras). The self-adjoint elements of the net are taken to represent localized physical quantities, while the Haag-Kastler axioms ensure that the assignment of quantities to regions obeys the joint requirements of relativity and quantum mechanics. ${ }^{10}$ Via the GNS theorem, each global state determines a unique representation of the net as bounded operators on a separable global Hilbert space, which in turn determines an expanded net of local von Neumann algebras, $\{\mathfrak{R}(O)\}$, and the global algebra, $\mathfrak{R}$, defined as the double commutant (or equivalently the weak-closure) of $\{\mathfrak{A}(O)\}$ and $\mathfrak{A}$ respectively in the given representation. This allows for the definition of additional representation-dependent observables including the Hamiltonian and superselected charges.

Von Neumann algebras are special $C^{*}$-algebras that contain a complete set of projection operators. They can be divided into three types based on the structure of their lattice of projections. Algebraically speaking, a projection is an element $E \in \mathfrak{R}$ such that $E^{2}=E$. Geometrically speaking, projection operators correspond to orthogonal projections onto closed subspaces $E \mathcal{H} \subseteq \mathcal{H}$. For our purposes we will only need a few basic facts. Type $I$ algebras contain minimal projections called atoms which correspond to 1-dimensional subspaces of $\mathcal{H}$ and act as generators of the algebra. It is typically assumed that the local von Neumann algebras in QFT are factors, i.e., they do not contain any central elements, which commute with the entire algebra. ${ }^{11}$ A type I factor is always isomorphic to $B(\mathcal{H})$ for some Hilbert space $\mathcal{H}$. If $\mathcal{H}$ is finite-dimensionsional, then the factor is type $\mathrm{I}_{n}$. If $\mathcal{H}$

\footnotetext{
${ }^{10}$ The standard six axioms are isotony, microcausality, covariance, vacuum, spectrum, and weak additivity. Halvorson and Müger (2006) §2 contains a precise formulation of these axioms and a discussion of basic structural results including the Reeh-Schlieder theorem.

${ }^{11}$ While the physical motivation for this assumption is still unclear, nothing in the present discussion turns on it.
} 
is infinite-dimensional, the factor is type $\mathrm{I}_{\infty}$. Non-relativistic quantum mechanics employs type $\mathrm{I}_{n}$ algebras almost exclusively.

In contrast, the local von Neumann algebras in QFT are generically type III factors. These algebras are always isomorphic to some proper subalgebra of $B(\mathcal{H})$, where $\mathcal{H}$ is infinite-dimensional. Type III factors lack atoms entirely and have no pure, normal states. ${ }^{12}$ In all known rigorous models of QFT, the local algebras assigned to compact doublecone regions are type III. Moreover, general theorems due to Fredenhagen (1985) and Buchholz and Verch (1995) show that for QFTs with a well-defined ultraviolet scaling limit, doublecone algebras must be type III. $^{13}$

From a physical standpoint, the type III property reflects the high degree of entanglement displayed by the QFT vacuum and similar global states. As a consequence of the Reeh-Schlieder theorem, Halvorson and Clifton (2000) prove that any global state which is analytic for the energy is entangled across arbitrary spacelike separated regions. Such states encompass many (if not all) physically reasonable global states including the vacuum state as well as charged states described by DHR/BF superselection theory. (Informally, the analyticity requirement means that the energy cannot grow too rapidly as a function of field strength.) The Reeh-Schlieder theorem only depends on the presence of long-range, infrared correlations. In contrast, the type III property is thought to be an ultraviolet effect.

\footnotetext{
${ }^{12}$ Normal states are weak*-continuous and give rise to countably additive probability measures. They correspond to states which can be represented by density operators on the relevant GNS Hilbert space.

Type II factors are also characterized by lack of atoms and pure, normal states, but they are in many ways less pathological than type III algebras (e.g., it is possible to define a semi-finite trace). While these algebras have interesting applications in quantum statistical mechanics, they are of limited use in QFT.

${ }^{13} \mathrm{~A}$ doublecone is formed by the intersection of a past and future lightcone. Doublecones are compact and causally complete (i.e., $O=O^{\prime \prime}$ ). The algebras assigned to such regions play a central role in defining many important models of QFT. The scaling limit assumption requires the renormalization group flow to approach a conformal fixed point in the short-distance/highenergy limit. Fredenhagen secures this using the compact localization properties of Wightman field operators, while Buchholz and Verch employ the more general framework of scaling algebras. See Halvorson and Müger (2006), $§ 2.5$ and Haag (1996), Ch. 5.6 for an overview of these results.
} 
Extant proofs ruling out type I and II algebras all rely on short-distance scaling assumptions which appear to be ineliminable. ${ }^{14}$ While we expect widespread entanglement based on the Reeh-Schlieder theorem, the type III property indicates just how bad this entanglement is. Summers and Werner (1988) use it to show that every normal state maximally violates Bell's inequalities across spacelike tangent doublecones. Clifton and Halvorson (2001) deploy it to prove that no local operation can disentangle the local vacuum state from its environment. Both results mark a significant departure from the non-relativistic norm.

The type III property poses a severe problem for the equivalence thesis. Since the local algebras are not type I, they cannot be defined as $B\left(\mathcal{H}_{O}\right)$ for some local Hilbert space $\mathcal{H}_{O}$; they can only be concretely represented as a proper subalgebra. By itself, however, the tensor product decomposition $\mathcal{H}=\mathcal{H}_{O} \otimes \mathcal{H}_{O^{\prime}}$ does not suffice to pick out a privileged type III subalgebra of $\mathcal{H}_{O}$. At a minimum, some additional input besides the collection of system-environment decompositions is needed to recover the full structure of algebraic QFT.

In fact, the problem is much worse. An immediate corollary of Summers and Werner's results is that in any QFT with a well-defined ultraviolet scaling limit, the required system-environment decompositions do not exist:

No-Go Lemma 1. In any model of the Haag-Kastler axioms with a well-defined ultraviolet scaling limit, if the local doublecone algebras $\mathfrak{R}(O)$ are not type $I$, then then there is no pair of Hilbert spaces $\left(\mathcal{K}_{1}, \mathcal{K}_{2}\right)$ and an isomorphism $V: \mathcal{H} \rightarrow$ $\mathcal{K}_{1} \otimes \mathcal{K}_{2}$ such that $V^{*} \mathfrak{R}(O) V \subset B\left(\mathcal{K}_{1}\right) \otimes I$ with $V^{*} \mathfrak{R}\left(O^{\prime}\right) V \subset I \otimes B\left(\mathcal{K}_{2}\right) .{ }^{15}$

\footnotetext{
${ }^{14}$ The local algebras of unbounded spacelike wedges must be type III regardless of any ultraviolet scaling assumptions. This is usually proven using the technique of half-sided modular translations (Driessler 1977, Longo 1979). The proof that doublecone algebras are also type III, proceeds by showing that in the short distance scaling limit, the modular structure of the doublecone algebras $\mathfrak{R}(O)$ coincides with the modular structure of the wedge algebras $\mathfrak{R}(W)$, and thus $\mathfrak{R}(O)$ must also be type III. Since thermodynamically well-behaved QFTs are thought to satisfy the split property, however, there will always be a type I factor $\mathfrak{N}$ such that $\mathfrak{R}(O) \subset \mathfrak{N} \subset \mathfrak{R}(W)$. Hence it appears that some assumption about the ultraviolet scaling properties of the doublecone algebras is essential.

${ }^{15}$ Proof. This is a corollary of Summers and Werner (1988) Thm. 6.1 which shows that there are no normal product states across tangent doublecones $O_{1}$ and $O_{2}$ in the situation described
} 
Like proofs of the type III property, this no-go lemma relies on assumptions about the ultraviolet regime of QFT. This should give us pause. Wallace $(2006,2011)$ forcefully argues that when interpreting QFT we should be cautious of drawing metaphysical conclusions from the short-distance/high-energy aspects of the theory. He contends that QFTs like the standard model are best viewed as effective field theories, low-energy approximations of some unknown, underlying theory of quantum gravity. Given results in modern renormalization analysis, we have good reason to believe these approximations are largely insensitive to the details of the exact short-distance theory. Nonetheless, we should not trust effective theories in the ultraviolet limit where we suspect that they break down. Consequently, Wallace and Timpson urge that for all practical purposes we can ignore the complications created by non-type I algebras. ${ }^{16}$

Insofar as we are interested in the metaphysics of the actual world, I agree. Any physical results that hinge on the ultraviolet structure of QFT should be viewed as provisional guide, at best. But SSR has the potential to be a highly general ontology for quantum theories of all shapes and sizes. We have many reasons to explore the metaphysics of exact, non-approximate QFTs. For example, we might be interested in high-level metaphysical debates about things like quantities,

by the lemma. Since $\mathfrak{R}\left(O_{2}\right) \subset \mathfrak{R}\left(O_{1}^{\prime}\right)$, there can be no normal product states across $O_{1}$ and $O_{1}^{\prime}$ either. Two commuting von Neumann algebras acting on the same Hilbert space can be split iff there exist normal product states across them.

If a theory satisfies Haag duality, then $\mathfrak{R}\left(O^{\prime}\right)=\mathfrak{R}(O)^{\prime}$, and a more elementary algebraic argument argument can be given. For purposes of reductio, assume such an isomorphism exists. Since $V^{*} \mathfrak{R}(O) V \subset B\left(\mathcal{K}_{1}\right) \otimes I$, it follows that $\left(B\left(\mathcal{K}_{1}\right) \otimes I\right)^{\prime} \subset\left(V^{*} \mathfrak{R}(O) V\right)^{\prime}$. But $\left(B\left(\mathcal{K}_{1}\right) \otimes I\right)^{\prime}=$ $I \otimes B\left(\mathcal{K}_{2}\right)$ and $\left(V^{*} \mathfrak{R}(O) V\right)^{\prime}=V^{*} \mathfrak{R}(O)^{\prime} V$, so $I \otimes B\left(\mathcal{K}_{2}\right) \subset V^{*} \mathfrak{R}(O)^{\prime} V$. But by hypothesis $V^{*} \mathfrak{R}\left(O^{\prime}\right) V \subset I \otimes B\left(\mathcal{K}_{2}\right)$, and so by Haag duality, $V^{*} \mathfrak{R}(O)^{\prime} V=I \otimes B\left(\mathcal{K}_{2}\right)$. But this is impossible. By the Tomita-Takesaki theorem $\mathfrak{R}(O)$ and $\mathfrak{R}(O)^{\prime}$ must have the same type, so $V^{*} \mathfrak{R}(O)^{\prime} V$ cannot be type $\mathrm{I}$, whereas $I \otimes B\left(\mathcal{K}_{2}\right)$ is type $\mathrm{I}$.

${ }^{16}$ See p. 711, footnote 14 (also p. 301, footnote 13 in Wallace 2012). Note that in order for the local algebras to be finite as they suggest, we would have to treat both the ultraviolet and infrared cutoffs literally. If we only view the former as a real, physical cutoff (as Wallace 2011 proposes) then the local algebras must be infinite as a consequence of the Reeh-Schlieder theorem. This situation is of course compatible with type $\mathrm{I}_{\infty}$ local algebras. The problem with extending SSR to exact QFTs without an ultraviolet cutoff is not that the local algebras are infinite, it is that they are not type I. 
laws, and causation that turn on possible physics, not just actual physics. More narrowly, we might be interested in conceptual questions about the compatibility of relativity and quantum mechanics, which will require the detailed study of both exact and effective QFTs to fully answer. Even if the QFTs describing our world are effective, some theorists (e.g., Rivasseau 1991) argue that exact QFTs can provide a unifying, explanatory idealization akin to the thermodynamic limit in statistical mechanics. In addition, both the string world-sheet formalism and the AdS-CFT correspondence suggest that studying exact conformally invariant QFTs may help us to better understand the metaphysics of string theory. If it turns out that SSR is only compatible with effective QFTs, this would be an important and interesting caveat. Moreover, the status of the type III property in effective theories is still unresolved. We can wager that the details do not matter in the end, but we cannot be sure at this stage. All things considered, it would be far better to have a version of SSR that can be applied both to effective and exact models of QFT regardless of what type the local algebras turn out to be.

If we drop the standing idea that localization information is encoded by tensor products we might make some progress in this direction. Given a local state $\rho(O)$, the GNS construction generates a canonical representation of the local algebra $\mathfrak{A}(O)$ as bounded linear operators acting on the GNS Hilbert space $\mathcal{H}_{\rho(O)}$. Wallace and Timpson suggest that this GNS Hilbert space can serve as the local Hilbert space of the field in region $O$. If we could somehow identify $\mathcal{H}_{\rho(O)}$ with a proper subspace of the global Hilbert space $\mathcal{H}_{\rho}$, we might hope to eventually recover the net of observable algebras from the collection of local GNS subspaces.

As it turns out though, the so-called "local" GNS Hilbert space $\mathcal{H}_{\rho(O)}$ does not really deserve the title. It is actually the global Hilbert space in disguise:

No-Go Lemma 2. Given a net of $C^{*}$-algebras satisfying the Haag-Kastler axioms, let $\rho$ denote a global state (analytic in energy) on the quasilocal algebra $\mathfrak{A}$ and let $\rho(O)=\left.\rho\right|_{\mathfrak{A}(\mathcal{O})}$. Up to unitary equivalence, the local GNS Hilbert space $\mathcal{H}_{\rho(O)}$ is naturally isomorphic to the global GNS Hilbert space $\mathcal{H}_{\rho}$, i.e., there is a natural family of isomorphisms mapping $\mathcal{H}_{\rho(O)}$ onto $\mathcal{H}_{\rho}$, intertwining the actions 
of $\pi_{\rho(O)}(\mathfrak{A}(O))$ and $\pi_{\rho}(\mathfrak{A}(O)) \cdot{ }^{17}$

In general, given a $C^{*}$-subalgebra $\mathfrak{B} \subset \mathfrak{A}$ and a restricted state $\left.\rho\right|_{\mathfrak{B}}$ as inputs, the GNS construction yields a Hilbert space $\mathcal{H}_{\rho \mid \mathfrak{B}}$ that can be naturally identified with a $\mathbb{C}$-closed subspace of $\mathcal{H}_{\rho}$. The hitch is that if the vector implementing $\rho$ is cyclic for $\mathfrak{B}$, then this subspace is identical to $\mathcal{H}_{\rho}$. The Reeh-Schlieder theorem guarantees that this will be the case for any global state analytic for the energy. Thus there is no formal sense in which applying the GNS theorem to local inputs yields a subsector of the global Hilbert space. ${ }^{18}$

If we take the message of this second lemma to heart, we should dispense not only with tensor products, but the very idea of a local Hilbert space. There is no obvious structure in the standard algebraic formalism that can be coherently identified as the local Hilbert space associated with a given region of spacetime. Perhaps there is some other clever way to work the standard formalism into alignment with SSR, but taken together these results strongly suggest that a different approach is needed.

\footnotetext{
${ }^{17}$ Proof. Let $\hat{\rho}, \hat{\rho}(O)$ denote the cyclic vectors representing $\rho$ and $\rho(O)$ in the GNS representations $\left(\pi_{\rho}, \mathcal{H}_{\rho}\right)$ and $\left(\pi_{\rho(O)}, \mathcal{H}_{\rho(O)}\right)$ respectively. First we show that $\mathcal{H}_{\rho(O)}$ can be naturally identified with a $\mathbb{C}$-closed subspace of $\mathcal{H}_{\rho}$. Taking $\pi_{\rho}(\mathfrak{A}(\mathcal{O}))$ as a subalgebra of $B\left(\mathcal{H}_{\rho}\right)$, we consider the compression map $\kappa:\left.\pi_{\rho}(A) \rightarrow \pi_{\rho}(A)\right|_{\mathcal{K}}$, for all $A \in \pi_{\rho}(\mathfrak{A}(\mathcal{O}))$, where $\mathcal{K}:=\overline{\pi_{\rho}(\mathfrak{A}(\mathcal{O})) \hat{\rho}}$ (the overline denotes the norm closure). This map is natural in the following sense: the GNS representation is fixed (up to unitary equivalence) by $\rho$ and $\mathfrak{A}$, and $\mathcal{K}$ is a $\pi_{\rho}(\mathfrak{A}(\mathcal{O})$ )-invariant subspace of $\mathcal{H}_{\rho}$, thus $\kappa$ is fixed (up to unitary equivalence) by $\rho, \mathfrak{A}$, and $\mathfrak{A}(O)$. Since $\mathcal{K}$ is $\pi_{\rho}\left(\mathfrak{A}(\mathcal{O})\right.$ )-invariant, it follows that $\kappa \circ \pi_{\rho}$ is a representation of $\mathfrak{A}(\mathcal{O})$ on $\mathcal{K}$. Moreover, $\hat{\rho}$ a cyclic vector for $\left(\kappa \circ \pi_{\rho}, \mathcal{K}\right)$ and $\rho(O)=\rho \circ\left(\kappa \circ \pi_{\rho}\right)$. These two conditions define the local GNS representation $\left(\pi_{\rho(O)}, \mathcal{H}_{\rho(O)}\right)$ up to unitary equivalence (Kadison and Ringrose, 1997, Prop. 4.5.3). Thus there exists an isomorphism $U$ from $\mathcal{H}_{\rho(O)}$ onto $\mathcal{K}$ intertwining the actions of $\pi_{\rho(O)}(\mathfrak{A}(O))$ and $\pi_{\rho}(\mathfrak{A}(O))$.

Next, we show that in fact $\mathcal{K}=\mathcal{H}_{\rho}$. By the Reeh-Schlieder theorem, we obtain $\overline{\pi_{\rho}(\mathfrak{A}(\mathcal{O}))^{\prime \prime} \hat{\rho}}=$ $\mathcal{H}_{\rho}$. But if $\hat{\rho}$ is cyclic for $\pi_{\rho}(\mathfrak{A}(\mathcal{O}))^{\prime \prime}$, it is separating for $\pi_{\rho}(\mathfrak{A}(\mathcal{O}))^{\prime \prime \prime}=\pi_{\rho}(\mathfrak{A}(\mathcal{O}))^{\prime}$, and hence must also be cyclic for $\pi_{\rho}(\mathfrak{A}(\mathcal{O}))$. Therefore $\mathcal{K}=\overline{\pi_{\rho}(\mathfrak{A}(\mathcal{O})) \hat{\rho}}=\mathcal{H}_{\rho}$. It follows that the compression $\kappa$ is the identity map, and $\left(\pi_{\rho(O)}(\mathfrak{A}(O)), \mathcal{H}_{\rho(O)}\right)$ is unitarily equivalent to $\left(\pi_{\rho}(\mathfrak{A}(O)), \mathcal{H}_{\rho}\right)$. Although the isomorphism $U$ implementing this equivalence may not be unique, it is part of a family determined by the natural compression $\kappa$, and each such isomorphism maps $\mathcal{H}_{\rho(O)}$ onto $\mathcal{H}_{\rho}$.

${ }^{18}$ Results from the modular localization program (Brunetti et al. 2002) indicate that it is possible to naturally assign a $\mathbb{R}$-closed proper subspace of the global Hilbert space to each spacetime region. Since these subspaces are not $\mathbb{C}$-closed, however, they are not large enough to be interpreted as local statespaces.
} 


\section{Statespace Axioms for QFT}

In order to formalize a version of SSR compatible with type III local algebras, we have to rethink the relationship between states and observables familiar to us from non-relativistic quantum mechanics. Fortunately, algebraic QFT offers such a perspective. Rather than rays in a local Hilbert space, in algebraic QFT local states are viewed as functionals over the local algebras. Towards the end of their discussion, Wallace and Timpson suggest that we can adopt this algebraic perspective as a foundation for SSR: "one can even remain at the more abstract level, forego the representation theorems and just take the $C^{*}$-algebraic state itself as denoting the properties of a region" (p. 712). The idea is never fully developed, however, and given that the local algebraic state is defined in terms of the observables it looks like a non-starter. What can the algebraic state possibly be besides a list of expectation values for the local observables?

In spite of this, thinking about the local state in abstract algebraic terms actually gives us a way to reframe SSR to accommodate the type III property. The key is to shift attention from the local state to the collection of possible local states. The statespace, $\mathcal{S}(\mathfrak{A})$, of a $C^{*}$-algebra $\mathfrak{A}$, is defined as the collection of all positive, normalized, $\mathbb{C}$-valued linear functionals on $\mathfrak{A}$. While the statespace is not a Hilbert space, it has a rich geometry; it is not just a bare set of functionals.

First, $\mathcal{S}(\mathfrak{A})$ is a convex set. Pure states are extremal points, while each mixed state can be written as a convex linear combination of distinct pure states,

$$
\rho=\lambda \rho_{1}+(1-\lambda) \rho_{2}
$$

where $\lambda \in(0,1)$. Second, $\mathcal{S}(\mathfrak{A})$ also has an order structure inherited from the order structure on $\mathfrak{A}: \rho_{1} \geq \rho_{2}$ iff $\rho_{1}(A)-\rho_{2}(A) \geq 0$ for all self-adjoint $A \in \mathfrak{A}$ with positive spectrum. In addition, $\mathcal{S}(\mathfrak{A})$ is compact in the weak ${ }^{*}$-topology. ${ }^{19}$ For each self-adjoint operator $A \in \mathfrak{A}$ there is a weak*-continuous affine function,

\footnotetext{
${ }^{19}$ This is defined as the coarsest topology such that every element of $\mathcal{S}(\mathfrak{A})$ corresponds to a continuous map on $\mathfrak{A}$.
} 
$\hat{A}: \mathcal{S}(\mathfrak{A}) \rightarrow \mathbb{R}$, defined by setting

$$
\hat{A}(\rho)=\rho(A)
$$

for all $\rho \in \mathcal{S}(\mathfrak{A})$. Kadison (1951) proves that the mapping sending $A \mapsto \hat{A}$ is an isometric isomorphism. This fact is the foundation for the spectral calculus on $\mathfrak{A}$ and shows that the self-adjoint part of $\mathfrak{A}$ is determined by the affine, order, and topological structure of $\mathcal{S}(\mathfrak{A})$. If $\mathfrak{A}$ is a von Neumann algebra, we may also choose to restrict attention its normal statespace, the set of all weak*-continuous elements of $\mathcal{S}(\mathfrak{A})$. The normal statespace has similar convexity and spectral properties but different topological features. ${ }^{20}$

An instructive, easily visualizable example is given by a 2-level quantum system. The relevant von Neumann algebra is $M_{2}(\mathbb{C})$, the algebra of $2 \times 2$ matrices with complex entries. This is a type $I_{2}$ factor isomorphic to $B\left(\mathcal{H}_{2}\right)$. In this case, every state is normal, so the statespace and the normal statespace coincide. It is affinely isomorphic to a 3-dimensional Euclidean sphere with unit radius (i.e., a Euclidean 3-ball). Each state can be written as a positive trace-one matrix,

$$
\frac{1}{2}\left(\begin{array}{cc}
1+x & y+i z \\
y-i z & 1-x
\end{array}\right)
$$

where $(x, y, z)$ are the Cartesian coordinates of the corresponding point in sphere. ${ }^{21}$ Boundary points represent pure states and interior points represent mixed states. If $\rho$ is a statistical mixture of pure states $\rho_{1}$ and $\rho_{2}$, then $\rho$ lies on the line segment connecting boundary points $\rho_{1}$ and $\rho_{2}$. Using Kadison's mapping, self-adjoint

\footnotetext{
${ }^{20}$ Typically, the normal statespace will not be compact in any useful topology, and the norm topology plays a more prominent role than the weak*-topology in many structural theorems. Interestingly, the statespace of an arbitrary $C^{*}$-algebra, $\mathfrak{A}$, is affinely isomorphic to the normal statespace of its universal enveloping von Neumann algebra, $\mathfrak{A}^{* *}$, (Alfsen and Shultz 2001, Cor. 2.126). Viewed as such, it has additional properties (e.g., weak*-compactness) that generic normal statespaces lack. Hence, while a von Neumann algebra is a special case of a $C^{*}$-algebra, a $C^{*}$-statespace is more properly viewed as a special case of a normal statespace.

${ }^{21}$ See Alfsen and Shultz (2001), Thm. 4.4.
} 
elements of $M_{2}(\mathbb{C})$ correspond to bounded $\mathbb{R}$-valued affine maps on the ball that attain their maximum and minimum on antipodal points.

These observations raise the following question: if we take local states rather than observables as primitive, is it possible to reconstruct the local observables as an algebra of functionals on the local statespace? A reconstruction theorem due to Alfsen, Hanche-Olsen, and Shultz (1980) shows that the answer is yes. ${ }^{22}$ Geometrically, the spectral information encoded by Kadison's isomorphism appears in the orthogonality relations between different faces of the convex set $\mathcal{S}(\mathfrak{A})$. A face is a convex subset, $F \subseteq \mathcal{S}(\mathfrak{A})$, that contains any line segment in $\mathcal{S}(\mathfrak{A})$ with interior points in $F$. (This generalizes the concept of a face of a polygon to an arbitrary convex set.) A face is exposed relative to some topology if the face can be isolated by intersecting $\mathcal{S}(\mathfrak{A})$ with a supporting hyperplane closed in that topology.

There is a tight structural analogy between exposed faces of $\mathcal{S}(\mathfrak{A})$ and projection operators in $\mathfrak{A}$. In the special case of a von Neumann algebra, this analogy is perfect: the norm-exposed faces of the normal statespace generate an orthomodular lattice which is naturally isomorphic to the lattice of projection operators. Thus the quantum logic of projections is mirrored in the facial structure of statespace. ${ }^{23}$ In our 2-level example, the norm-exposed faces are just the boundary points of the 3-ball. Each such point is a pure state associated with a projection operator with expectation value one in that state. Orthogonal projection operators are associated with antipodal points.

In order to recover the full structure of the corresponding algebra, one additional piece of geometric information is needed, a statespace orientation. The smallest face of $\mathcal{S}(\mathfrak{A})$ containing a pair of distinct pure states is affinely isomorphic to either a straight line or a Euclidean 3-ball. (The face is a line iff the GNS representations of the associated states are quasiequivalent and a 3-ball iff

\footnotetext{
${ }^{22}$ Alfsen and Shultz $(2001,2003)$ give a comprehensive treatment of these results.

${ }^{23}$ The general case is a bit more complicated since $C^{*}$-algebras do not always possess a complete set of projections, but the basic idea is the same. In full generality, the lattice of weak*-semiexposed faces in $\mathcal{S}(\mathfrak{A})$ is isomorphic to the lattice of upper semi-continuous projections in the universal enveloping algebra $\mathfrak{A}^{* *}$ (Alfsen and Shultz 2001, Thm. 3.61).
} 
the representations are disjoint.) The non-commutative operator product on $\mathfrak{A}$ determines an orientation for each facial 3-ball given by an equivalence class of affine automorphisms where two automorphisms are equivalent just in case they can be related by a rotation. ${ }^{24}$ Alfsen et al. (1980) prove the converse, showing that a suitably continuous choice of orientation for each facial 3-ball suffices to determine the operator product of the associated algebra.

Geometrically, choosing an orientation determines a unique correspondence between $\mathbb{R}$-valued affine functions on $\mathcal{S}(\mathfrak{A})$ (i.e., self-adjoint observables) and 1parameter groups of affine automorphisms of $\mathcal{S}(\mathfrak{A})$. From a physical standpoint, this correspondence captures the role that observables play as infinitesimal generators of particular symmetries. The 2-level example elegantly illustrates this idea. Each self-adjoint $A \in M_{2}(\mathbb{C})$ acts as a $\mathbb{R}$-valued functional on the the Euclidean 3-ball, attaining a maximum and minimum value on a pair of antipodal points. The operators $i A$ and $-i A$ generate infinitesimal statespace symmetries, rotations of the 3-ball around the diameter between these points. The choice of an orientation structure determines which of these generate clockwise and counterclockwise rotations.

The AHS-reconstruction theorem shows that it is possible to recover a local observable algebra from its oriented statespace. This is not enough to capture the full physics of QFT, however, since in most models the local algebras are isomorphic. Consequently the physical differences between various models of QFT must be encoded in the relations between local algebras rather than their internal algebraic structure. We must find a way to recover the structure of the complete net of observable algebras given suitable relations between their statespaces.

The eventual goal is to formulate extensionally equivalent statespace analogues of the standard Haag-Kastler axioms. The first axiom, isotony, requires that if $O_{1}$

\footnotetext{
${ }^{24}$ For each facial 3-ball, this effectively corresponds to the usual notion of orientation for a manifold. In particular, a 3-ball always admits two possible orientations, "right" and "left" handed. This is not true across the entire statespace. In general there will be infinitely many suitably continuous ways to choose an orientation for each facial 3-ball corresponding to the infinite number of Lie products compatible with the Jordan product on $\mathfrak{A}$.
} 
is a subregion of $O_{2}$, the local algebra $\mathfrak{A}\left(O_{1}\right)$ must be a subalgebra of $\mathfrak{A}\left(O_{2}\right)$. This gives the collection of local algebras the structure of a net, and allows the quasilocal algebra to be defined as the colimit of this structure. Its dual, $\mathcal{S}$-isotony requires the existence of a privileged restriction mapping, $\psi: \mathcal{S}\left(O_{2}\right) \rightarrow \mathcal{S}\left(O_{1}\right)$, between the corresponding oriented local statespaces. Formally, $\psi$ must be an $\mathcal{S}$-homomorphism, a weak ${ }^{*}$-continuous affine surjection which preserves complementary faces and whose inverse preserves orientation. This gives the collection of local statespaces the structure of a presheaf, dual to the net of observable algebras, and allows us to define the quasilocal statespace as its limit.

The translation of the remaining axioms is somewhat mathematically involved, but the basic motivation is straightforward. $\mathcal{S}$-microcausality says that the exposed faces of spacelike separated local statespaces must be antipodal (except possibly on their intersection) when embedded within the statespace of any enveloping region. This mirrors the idea that two algebras commute iff their associated spectral projections do. $\mathcal{S}$-covariance requires the existence of a suitably continuous representation of the Poincare group (or the translation subgroup) in the group of automorphisms of the presheaf of local statespaces. This encodes the dynamics and allows us to identify the vacuum state as a fixed point of the group action. The $\mathcal{S}$-analogue of the spectrum condition requires that the vacuum state lie in the (exposed, split) face generated by the positive energy states (i.e., those states whose Fourier transformations have particular spectral support properties relative to the momentum-space forward lightcone).

Drawing upon these ideas, we can frame a revised version of Wallace's equivalence thesis:

Revised Equivalence Thesis. Given an assignment, $\{\mathcal{S}(O)\}$, of oriented statespaces to spacetime regions satisfying the $\mathcal{S}$-axioms, we can define a dual net of observables satisfying the Haag-Kastler axioms. Moreover, any net satisfying the usual axioms arises in this way.

This gives us sharpened mathematical conjecture that has a fighting chance of actually being true. The $\mathcal{S}$-axioms characterize the family of mappings between 
local statespaces missing from Wallace's original version. Moreover, because of the 1-1 correspondence between oriented statespaces and $C^{*}$-algebras given by the AHS-reconstruction theorem, the revised thesis is compatible with local algebras of any type. It stands to provide a state-first formulation of QFT that has the same flexibility and power as algebraic QFT.

A follow-up paper, Swanson and Halvorson (in preparation), will provide a mathematically precise formulation of the $\mathcal{S}$-axioms and endeavour to prove the revised equivalence thesis. The primary goal here has been to motivate the kind of formal framework needed to circumvent the type III problem. Our conceptual understanding of QFT has been greatly enhanced by shifting to an abstract algebraic picture on the observable side. It is only natural to suspect that the parallel move on the state side will provide similar insight into the foundations of relativistic SSR. To assuage the skeptical reader, we note that Shultz (1981) proves that $\mathcal{S}$-homomorphisms are dual to $*$-homomorphisms, establishing that the AHS-reconstruction result is categorical. Thus from a purely mathematical angle, anything we can do with $C^{*}$-algebras, we can do with oriented statespaces. It remains to be seen if the revised equivalence thesis is similarly categorical and what independent physical motivation can be given for the $\mathcal{S}$-axioms. ${ }^{25}$

The defender of SSR thus has two options on the table. She can restrict the scope of her thesis to effective QFTs and use Wallace and Timpson's original proposal, or she can broaden her sights and pursue the statespace approach advocated here. The flexibility to treat non-type I local systems is a marked advantage of the latter. In addition, the dual statespace language facilitates connections with the extensive literature on algebraic and constructive QFT. In many instances we

\footnotetext{
${ }^{25}$ In addition, we might eventually hope to develop statespace analogues of standard quantization techniques. As long as our methods for constructing QFTs rely on operators as tools, there will be the worry that the statespace framework is not sufficiently independent. The relationship between the new framework and constructive QFT is an important issue, but one beyond the scope of this paper. Landsman (1998), Ch. 1.2-1.3 develops a geometric quantization procedure linking the pure statespace of a type I quantum mechanical system to a classical Poisson manifold where both are viewed as transition probability spaces. These techniques could provide a bridge to more general statespace quantization procedures.
} 
can appeal to the duality between algebras and statespaces to show that a certain construction must be possible on the statespace side, even if we do not yet know all of the details. In the final section, we will use this technique to diffuse a number of preliminary objections that have been levied against SSR.

\section{Discussion}

SSR claims that the local state encodes intrinsic properties of a region, but what exactly are these properties? Wallace and Timpson recognize that they must be "admittedly somewhat alien," but urge that the situation is in principle no different from classical field theories: "it is not as if we really have an intuitive grasp of what an electric or a magnetic field is, other than indirectly and by means of instrumental considerations ('A test charge would be accelerated thus,' for example)" (p. 699-700). They argue that everything must ultimately be analyzed in terms of patterns of fundamental properties of spacetime regions, and that our epistemic grip on the properties themselves comes from two sources: (1) the structure of the mathematical entities used to represent them, and (2) instrumental connections between these entities and observation. As critics have noted, however, there are serious concerns about both (1) and (2).

One prominent objection to SSR is that we do not adequately understand the relevant instrumental connections (2). Both Lewis (2013) and Baker (2016) worry that the view makes the relationship between fundamental quantities and experimental predictions hopelessly obscure, if not incoherent. Lewis observes that density operators are represented by complex matrices, "but the matrix elements themselves determine a probability distribution over outcomes. How do the actual outcomes give us insight into probabilistic properties?" As Baker puts it,

We derive statistical predictions from the quantum state via expectation values, but what is the meaning of an expectation value of energy, for example, if energy is not among the fundamental quantities (which are exhausted by the local states)? Moreover, how are we to make 
sense of a deterministic theory whose fundamental quantities appear to be specified indirectly by way of statistical expectation values?

Baker goes on to point out that Wallace and Timpson criticize rival views on the grounds that they posit a brute connection between operators and expectation values, rather than providing an explanation of how expectation values arise through an analysis of the physics of measurement. But according to Baker, essentially the same criticism applies to SSR: the fundamental quantities, the local states, directly encode expectation values by fiat.

While these objections target (2), at their core lies a host of confusions surrounding (1), most conspicuously, the idea that the local state is nothing more than a list of expectation values. The defender of SSR should reject this idea (as Wallace and Timpson do), but it is incredibly difficult to see how this is possible from the perspective of the original density operator formalism.

The difficulty is brought out by a pair of puzzles about the symmetries of Hilbert space that cast doubt on whether the field of local density operators is sufficient to encode all of the relevant physics. Wallace (2012) observes that we usually think of the quantum state as represented by a ray rotating in Hilbert space. But Hilbert spaces are highly symmetrical (up to isomorphism they are only distinguished by dimension). Each ray is just like every other, so it is not clear how a ray can represent a structured entity like the quantum state in a region. A second, related puzzle, originally put to me by Baker (personal communication, February 2013), turns on essentially the same technical point. All existing models of QFT employ infinite-dimensional, separable Hilbert spaces as the global statespace, and all such Hilbert spaces are isomorphic. Therefore by Gleason's theorem, all models of QFT have the same set of global density operators. These in turn determine the local density operators. But if this is all there is to the ontology of QFT, as SSR suggests, then it seems that SSR cannot distinguish between physically inequivalent models of QFT, e.g., a free Klein-Gordon model and an interacting $\phi^{4}$-model.

In response to the first puzzle, Wallace concludes that in addition to density 
operators we need to specify a privileged set of observables acting on the Hilbert space. This breaks the symmetry, allowing different rays to represent distinct physical possibilities: "Differences between states correspond to differing patterns of assignments of numbers to operators, and those patterns can be highly structured" (p. 296). A similar response can be given to the second puzzle: although the Klein-Gordon model and the $\phi^{4}$-model are implemented on isomorphic Hilbert spaces, they carry unitarily inequivalent representations of the canonical commutation relations which breaks the symmetry. The patterned assignment of numbers to operators is different in the free and interacting theory. Yet even if we treat the operators as merely formal tools as Wallace and Timpson urge, it remains unclear how we should interpret the numbers assigned to them by the states. If they represent expectation values, then the Lewis-Baker objection seems on point differences between states are differences in fundamentally probabilistic properties. If they represent something else, then what, and why are these entities directly correlated with expectation values?

The equivalence thesis suggests a possible escape route. All of this algebraic information can instead be encoded in the localization structure, in the tensor product decomposition of the global Hilbert space into subsystems. A state is not just a ray or a density operator, but rather a section of this tensor product structure. Differences between theories boil down to differences in their subsystem decompositions. Although patterns of expectation values can reveal the complexity of this geometry, these patterns emerge from the localization structure, not vice versa.

Unfortunately, in its original formulation the equivalence thesis is far too vague to be of much practical help. As we have seen, most of the physical content is actually contained in mappings between different tensor product decompositions, however the thesis is largely silent on what this network of mappings should look like. It is not clear at this stage how to identify SSR field configurations as sections of some suitable bundle-like structure, nor is it clear how differences between unitarily inequivalent QFTs can be grounded in different tensor product decom- 
positions. Here, the new statespace formulation of SSR can be of great service. It offers a rich geometry capable in principle of encoding everything that can be found in algebraic QFT. Three basic observations about this geometry serve to dissolve the two symmetry puzzles and counter the Lewis-Baker objection.

First, the $\mathcal{S}$-isotony and $\mathcal{S}$-microcausality axioms provide a more precise characterization of the localization structure in QFT than Wallace's vague suggestions about tensor product decompositions. The antipodality constraints imposed by $\mathcal{S}$ microcausality ensure that spacelike separated systems are suitably independent, while the network of restriction mappings characterized by $\mathcal{S}$-isotony provides information about the relations between regions, weaving the collection of local statespaces together into a single mathematical object whose sections represent SSR field configurations. It also ensures that each point in the statespace associated with region $O$ has a canonical restriction to a unique point in the statespace of each subregion. Thus we can speak coherently of the local presheaf associated with each region. The local state is not just a point in the convex set $\mathcal{S}(O)$, it is a section of the local presheaf. Points which are intrinsically alike with respect to the geometry of $\mathcal{S}(O)$ can correspond to geometrically distinct sections of the local presheaf.

Second, by itself, this presheaf structure is insufficient to do physics. Unitarily inequivalent QFTs like the Klein-Gordon model and $\phi^{4}$-model or Klein-Gordon models with different masses can be constructed by starting with isomorphic nets of $C^{*}$-algebras. They are differentiated by choosing inequivalent representations of the Poincaré group acting as automorphisms of the net. In suitable positiveenergy GNS representations, privileged sets of operators are singled out as generators of the corresponding spacetime symmetries, justifying their role as energymomentum and angular-momentum observables. In Wallace's picture, these observables serve a critical function, essentially labeling sectors of Hilbert space so that otherwise identical rays can encode states with different physical content. In our revised statespace picture, the $\mathcal{S}$-covariance axiom specifies a representation of the Poincaré transformations acting as 1-parameter groups of presheaf auto- 
morphisms. The orbits of these groups, rather than a set of energy-momentum observables, serve to label portions of statespace so that otherwise identical sections can represent different configurations of the state-valued field.

Third, given a net of $C^{*}$-algebras equipped with a representation of the Poincaré group, it remains possible to choose different global states and, via the GNS construction, arrive at unitarily inequivalent representations of the net. Such representations can be distinguished by different global boundary conditions characterized by different global von Neumann algebras. This scenario arises, for instance, when describing different charge sectors in theories characterized by DHR/BF superselection theory. The global algebras in each sector contain non-trivial central elements that are the spectral projections of associated charge observables. These structural differences are mirrored in the convex geometry of the dual global statespaces (where central projections correspond to split faces). Like algebraic QFT, the statespace version of SSR thus gives us the flexibility to handle reducible representations with global algebras other than $B(\mathcal{H})$.

To sum up: a state is not just a ray or density operator, nor is is just a point in a convex, oriented set. It is a section of a presheaf of such sets, carrying a privileged representation of the Poincaré group, and thus a highly structured entity. This geometry is dual to a net of $C^{*}$-algebras, and provided that the revised equivalence thesis is correct, it gives us all of the necessary tools to individuate unitarily inequivalent models of QFT. Differences between models boil down to differences in the presheaf structure, the Poincaré representation, or the structure of the global statespace in particular representations. These observations also serve to clarify Wallace's conjecture that the localization structure is sufficient to determine the physics. If "localization structure" is interpreted to mean a presheaf of oriented $C^{*}$-statespaces satisfying $\mathcal{S}$-isotony and $\mathcal{S}$-microcausality, then the conjecture is false. In addition to knowing which states are localized in which regions, we need to know which symmetries of the presheaf correspond to which spacetime symmetries and which global states are physically possible. ${ }^{26}$ But SSR does not sink with

\footnotetext{
${ }^{26}$ Is this enough? That remains to be seen. If unitary equivalence is sufficient for physical
} 
this conjecture (whose primary purpose was to help make the equivalence thesis plausible). Rather, we have simply gained a better understanding of the convex geometry that characterizes the field of local states. ${ }^{27}$

By clearly separating this geometry from talk about "assignments of numbers to operators," the view also provides more breathing room to begin responding to Lewis and Baker. At the fundamental level, the field dynamics are completely deterministic. There are no primitive probabilistic properties (unless we modify the theory in the service of a collapse interpretation). We can introduce observables as bounded affine functions on the presheaf of local statespaces, but although their

equivalence, then it should be. Much of the algebraic QFT literature assumes that physical differences between models supervene on the net of $C^{*}$-algebras, the Poincaré representation, and the set of physically possible global states. Once these structures are fixed, we do not need to be told which operators represent which physical quantities. This can be inferred from the kinematical and dynamical properties of the model. SSR adopts the same mantra on the statespace side. If it turns out that quantization rules or some other physical correspondence principles are essential not just for constructing models but for endowing them with their physical content, then more might be needed. The aim here is not the settle this question decisively, but the put SSR on a formally coherent foundation, so that its merits and flaws can be better assessed.

A further point of clarification: if "localization structure" is instead interpreted to mean a presheaf of statespaces dual the the net of von Neumann algebras in a particular representation, then the status of Wallace's localization conjecture is less clear. The global von Neumann algebra now appears explicitly as part of the presheaf structure, and algebraic proofs of the PCT and spinstatistics theorems indicate that we can naturally reconstruct a representation of the Poincaré group from a net of concrete von Neumann algebras satisfying microcausality and a further technical condition, modular covariance (Guido and Longo 1995).

It is doubtful that this move plausibly captures Wallace's original intuition, however. Modular covariance requires that certain algebraic invariants (the modular automorphism groups associated with wedge-localized von Neumann algebras) generate certain spacetime symmetries. Thus we still need information relating symmetries of the net of to symmetries of spacetime, only now this information is encoded in the algebraic properties of an enriched net of operators. Many of these extra operators, including the modular invariants, are not observables at all. It seems unlikely that this constraint can be plausibly interpreted as an assumption about the localization of physical quantities.

${ }^{27}$ It might be objected that the current proposal, which posits geometric relations between local states, is less ontologically parsimonious than the original version of SSR, which simply posits an assignment of states to regions. It should be noted, however, that the original proposal already posits substantial geometric relations between local states encoded in the Hilbert space and tensor-product structure (e.g., convexity, extendability, and orthogonality relations). It is true that additional geometric relations are needed, but without them SSR will not be capable of adequately differentiating models of QFT. 
mathematical structure is fixed once the statespace structure is specified, their physical interpretation is not. Which functions encode the expectation values for which kinds of measurements must be explained. One possible strategy would analyze measurement procedures in terms of functional patterns in the state-valued field configuration. (The details would depend on how we choose to solve the measurement problem.) Particular affine functions would then be identified with equivalence classes of measurement procedures. If successful, this strategy would in some sense vindicate the early operationalist interpretation of observables in algebraic QFT. ${ }^{28}$ The crucial difference is that it would do so by providing a realist explication of concepts like "measurement device" and "preparation procedure," concepts taken as primitive by the operationalist view. Differences between states ultimately give rise to different patterns of expectation values, but only after a long, complex physical explanation. The states do not encode expectation values by fiat. In fact, it is the observables which emerge as lists of expectation values on this view. ${ }^{29}$

Obviously, much work remains to be done in order to spell out the details of such a story. There may be other viable strategies as well. Baker (2016) is right to point out that "a perspicuous explanation for the expectation values is a major missing piece in extant field interpretations" like SSR. But by recasting the quantum state as a section of a highly structured presheaf, we can start to see how such an explanation might be possible, as well as some of the broad contours it could take. (For this reason, the present proposal should be of interest not just

\footnotetext{
${ }^{28}$ See Araki (1999), Ch. 1 for a summary of the central motivating ideas.

${ }^{29}$ To be clear, the view is not that physical quantities, such as energy, are equivalence classes of measurement procedures, only that the corresponding observables are. The state-valued SSR field is a physical field, and just like the tensor-valued electromagnetic field, facts about its energy content will supervene on facts about the underlying field configuration. If this is right, we should be able to identify some structural pattern in the SSR field as its energy (similar to the definition of the electromagnetic stress energy-tensor in terms of the electromagnetic field tensor). The energy observable, an $\mathbb{R}$-valued functional over the SSR field, is interpreted operationally as a list of expectation values associated with a suitable equivalence class of measurement procedures that reliably track information about the energy content of the SSR-field. In short, observables no longer directly represent physical quantities as they do on the usual interpretation of algebraic QFT.
} 
to philosophers concerned with the technical foundations of QFT, but to those interested in non-relativistic quantum theory as well.) The ultimate viability of relativistic SSR, including its relationship to more traditional interpretations of algebraic QFT, hinges on a proof of the revised equivalence thesis and a detailed study of the $\mathcal{S}$-axioms. Only this will reveal whether or not the state-valued field is sufficiently structured to ground the kind of physical emergence story that SSR wants to tell. By framing the thesis as a precise mathematical conjecture and providing the tools to solve the type III problem, the preceding investigation gives us the roadmap necessary to begin this project in earnest.

\section{Acknowledgements}

This paper originated in a series of fruitful conversations with David Baker and Hans Halvorson, who challenged me to explore the difficulties involved in recovering algebraic QFT from SSR. I would also like to thank David Wallace, Christopher Timpson, David Albert, Gordon Belot, Laura Ruetsche, and two anonymous referees, along with audiences at Columbia, Rutgers, Michigan, and Rome Tor Vergata for helpful comments and criticism on early drafts of this paper. This work was supported in part by University of Delaware GUR Grant No. 15A00707.

\section{References}

Alfsen, E., Hanche-Olsen, H., and Shultz, F. (1980). State spaces of $C^{*}$-algebras. Acta Mathematica, 144:267-305.

Alfsen, E. and Shultz, F. (2001). State Spaces of Operator Algebras. Birkhäuser.

Alfsen, E. and Shultz, F. (2003). Geometry of State Spaces of Operator Algebras. Birkhäuser.

Araki, H. (1999). Mathematical Theory of Quantum Fields. Oxford UP, Oxford. Arntzenius, F. (2012). Space, Time, and Stuff. Oxford University Press. 
Baker, D. J. (2016). The philosophy of quantum field theory. In Oxford Handbooks Online. Oxford University Press.

Brunetti, R., Guido, D., and Longo, R. (2002). Modular localization and Wigner particles. Reviews in Mathematical Physics, 14:759-785.

Buchholz, D. and Verch, R. (1995). Scaling algebras and renormalization group in algebraic quantum field theory. Reviews in Mathematical Physics, 7:1195.

Clifton, R. and Halvorson, H. (2001). Entanglement and open systems in quantum field theory. Studies in History and Philosophy of Modern Physics, 32:1-31.

Driessler, W. (1977). On the type of local algebras in quantum field theory. Communications in Mathematical Physics, 53:295-297.

Fredenhagen, K. (1985). On the modular structure of local algebras of observables. Communications in Mathematical Physics, 97:79-89.

Guido, D. and Longo, R. (1995). An algebraic spin statistics theorem. Communications in Mathematical Physics, 172:517-533.

Haag, R. (1996). Local Quantum Physics. Springer-Verlag, Berlin.

Halvorson, H. and Clifton, R. (2000). Generic Bell correlations between arbitrary local algebras in quantum field theory. Journal of Mathematical Physics, 41(4):1711-1717.

Halvorson, H. and Müger, M. (2006). Algebraic quantum field theory. In Butterfield, J. and Earman, J., editors, Philosophy of Physics, pages 731-922. Elsevier.

Ismael, J. and Schaffer, J. (2016). Quantum holism: nonseparability as common ground. Synthese.

Kadison, R. V. (1951). A representation theory for commutative topological algebra. Memoirs of the American Mathematical Society, 7. 
Kadison, R. V. and Ringrose, J. R. (1997). Fundamentals of the Theory of Operator Algebras. Vol. I \& II. American Mathematical Society, Providence, RI.

Landsman, N. (1998). Mathematical Topics Between Classical and Quantum Mechanics. Springer-Verlag, New York.

Lewis, P. (2013). Review of: The Emergent Multiverse. Notre Dame Philosophical Reviews.

Longo, R. (1979). Notes on algebraic invariants for non-commutative dynamical systems. Communications in Mathematical Physics, 69:195-207.

Rivasseau, V. (1991). From Perturbative to Constructive Renormalization. Princeton University Press.

Shultz, F. (1981). Dual maps of Jordan homomorphisms and $*$-homomorphisms between $C^{*}$-algebras. Pacific Journal of Mathematics, 93(2):435-441.

Summers, S. J. and Werner, R. (1988). Maximal violations of Bell's inequalities for algebras of observables in tangent spacetime regions. Annales de l'Institute Henri Poincaré. Physique Théorique, 49(2):215-243.

Swanson, N. and Halvorson, H. (in preparation). Statespace axioms for QFT.

Wallace, D. (2006). In defence of naiveté: On the conceptual status of Lagrangian quantum field theory. Synthese, 151:33-80.

Wallace, D. (2011). Taking particle physics seriously: A critique of the algebraic approach to quantum field theory. Studies in History and Philosophy of Modern Physics, 42:116-125.

Wallace, D. (2012). The Emergent Multiverse. Oxford University Press.

Wallace, D. (2013). A prolegomenon to the ontology of the Everett interpretation. In Ney, A. and Albert, D., editors, The Wavefunction: Essays in the Metaphysics of Quantum Mechanics. Oxford University Press. 
Wallace, D. and Timpson, C. G. (2010). Quantum mechanics on spacetime I: Spacetime state realism. British Journal for the Philosophy of Science, 61:697727. 